\title{
VECTOR INTERPRETATION OF SYMBOLIC DIFFERENTIAL
}

\section{PARAMETERS *}

BY

\section{LOUIS INGOLD}

\section{INTRODUCTION.}

The purpose of this paper is to establish a relation between the symbolic theory of invariants of differential forms, due to the late Professor MaschKe, $\dagger$ and the theory of extensive quantity (vectors), due to Grassmann. $t$

It will be shown that those symbolic expressions used by MASCHKE which lacked an interpretation in his theory, may be represented as vectors of the Grassmann type, and that all of MaschKe's expressions, including his actual differential parameters, are expressible in the vector system. The theory of such vectors will be extended, new formulas in the symbolic theory will be obtained and applications to geometry will be made.

A preliminary section on space of two dimensions is given, in order that the well known geometric representation of vectors in two dimensions may serve as a basis for a compact treatment of the general theory.

The general theory is stated for three dimensions in concise form, proofs being omitted when their details are simple extensions of the two dimensional case; the obvious generalization to Euclidean space of $n$ dimensions is then indicated.

The less obvious extension to surfaces in three dimensions and, generally, to arbitrary $k$-dimensional spaces imbedded in an Euclidean space of $n$ dimensions is treated in more detail. This involves an application of the GrassmanN theory to non-uniform spaces and furnishes an example of a vector system based on variable units.

The paper closes with a section dealing with differential parameters which involve second and higher derivatives of the symbols.

* Presented to the Society (Chicago), March 30, 1907, in somewhat different form, under the title: Vector theory, in terms of symbolic differential parameters.

† MAsCHKE, $A$ Symbolic Treatment of the Theory of Invariants of Quadratic Differential Quantics of $n$ Variables, Transactions of the American Mathematical Society, vol. 4 (1903), pp. 445-469.

† H. Grassmand, Ausdelinungslehre, 1862. References are to Grassmann's collected works edited by F. ENGKL. 


\section{The Two-dimensional Case.}

1. Comparison of the properties of vectors and symbols. Given any binary quadratic differential form,

$$
A \equiv E(x, y) d x^{2}+2 F(x, y) d x d y+G(x, y) d y^{2},
$$

Maschke replaces the form by a symbolic square

$$
A \equiv\left[f_{(1)}(x, y) d x+f_{(2)}(x, y) d y\right]^{2 *}
$$

and writes symbolically

(3) $f_{(1)}^{2}(x, y)=E(x, y) ; f_{(1)}(x, y) f_{(2)}(x, y)=F(x, y) ; f_{(2)}^{2}(x, y)=G(x, y)$.

In the special case $E=G=1, F=0$, the differential form represents the square of the element of length in a plane; we have then

$$
A=d s^{2}=d x^{2}+d y^{2}=\left(f_{(1)} d x+f_{(2)} d y\right)^{2},
$$

where $f_{(1)}$ and $f_{(2)}$ are constant, and where

$$
f_{(1)}^{2}=1, \quad f_{(1)} f_{(2)}=0, \quad f_{(2)}^{2}=1 .
$$

The formulas (5) suggest the properties of so-called inner multiplication of a set of unit vectors in a plane. Thus if $e_{1}$ and $e_{2}$ are unit vectors along the $x$ and the $y$-axis respectively, and if $a=a_{1} e_{1}+a_{2} e_{2}$ and $b=b_{1} e_{1}+b_{2} e_{2}$ are any vectors, the inner product is

$$
[a \mid b]=a_{1} b_{1}+a_{2} b_{2} \cdot \dagger
$$

Then for inner multiplication

$$
\left[e_{1} \mid e_{1}\right]=e_{1}^{2}=1, \quad\left[e_{2} \mid e_{2}\right]=e_{2}^{2}=1, \quad\left[e_{1} \mid e_{2}\right]=\left[e_{2} \mid e_{1}\right]=0 .
$$

On account of this agreement, the Maschke symbols $f_{(1)}$ and $f_{(2)}$ may be

* MAschKe, loo. oit., p. 448.

The general quadratio differential form

$$
\sum_{i, j=1}^{n} a_{j} d x_{i} d x_{j}
$$

in $n$ variables $x_{1}, x_{2}, \cdots, x_{n}$ is replaced by $\left[f_{(1)} d x_{1}+f_{(2)} d x_{2}+\cdots+f_{(n)} d x_{n}\right]^{2}$, so that symbolically $f_{(i)} f(j)=a_{i j}$; for the special differential form

these equations become

$$
\sum_{i=1}^{n} d x_{i}^{2}
$$

$$
f(i) f(j)=\left\{\begin{array}{l}
0, i+j \\
1, i=j .
\end{array}\right.
$$

†The inner product is also defined to be $a b \cos a \hat{b}$, where $a$ means the length of $a, i$. e., $\sqrt{a_{1}^{2}+a_{2}^{2}}, b=\sqrt{b_{1}^{2}+b_{2}^{2}}$, and $\hat{a b}$ is the angle between $a$ and $b$. This definition is equivalent to the one given in the text. 
thought of as a set of unit vectors $\boldsymbol{e}_{1}$ and $\boldsymbol{e}_{2}$, the multiplications in (5) being inner multiplications.

It will be shown later that this correspondence of properties persists between the Maschke symbols and the vectors of Grassmann's theory in space of $n$ dimensions, every essential property being identical in the two cases.

We proceed just now to show that many - in fact all - of the various symbolic expressions used by Maschke for two dimensions have a meaning in the vector theory; if we set $f_{(1)}=e_{1}$ and $f_{(2)}=e_{2}$ as suggested above; and that the relations existing between his symbolic expressions correspond to geometric relations.

2. Notations. It is found convenient in the synbolic theory to introduce other symbols, $\phi_{(1)}, \phi_{(2)}$ equivalent to $f_{(1)}, f_{(2)}$, in the sense that

$$
\phi_{(1)}^{2}=E, \quad \phi_{(1)} \phi_{(2)}=F, \quad \phi_{(2)}^{2}=G,
$$

according to equations (3). With the aid of two sets of symbols, $f_{(1)}, f_{(2)} ; \phi_{(1)}, \phi_{(2)}$, expressions quadratic in the $E, F, G$ may be represented symbolically; thus, $E^{2}=f_{(1)}^{2} \phi_{(1)}^{2}, \quad E F=f_{(1)}^{2} \phi_{(1)} \phi_{(2)}, 2\left(E G-F^{2}\right)=\left(f_{(1)} \phi_{(2)}-f_{(2)} \phi_{(1)}\right)^{2}, \quad$ etc.

When convenient, still other symbols are introduced, equivalent to $f$ and $\phi$, in the sense described above. It is agreed, however, that no more than two symbols of the same kind shall occur in the same product, and that in every product two symbols of like kind, i. e., two $f$ 's or two $\phi$ 's, etc., occurring as factors are to be interpreted according to equations (3) [or equations (5) in the special case we are now considering]. Otherwise the symbols are subject to the same laws as ordinary algebraic quantities. The discriminant $E G-F^{2}$ of the general quadratic form (1) is denoted by $1 / \beta^{2}$.* In general, $\beta$ is a function of $x$ and $y$; but $\beta=1$ for the special differential form (4).

The symbols $f_{(1)}, f_{(2)}$ were regarded by Maschke as partial derivatives of a symbolic function $f(x, y)$, so that $f_{(1)}=\partial f / \partial x, f_{(2)}=\partial f / \partial y$.

In the special case $(4)$, since $f_{(1)}$ and $f_{(2)}$ are constant we may write $f=f_{(1)} x+f_{(2)} y$.

The Jacobian

$$
\begin{array}{ll}
U_{(1)} & V_{(1)} \\
U_{(2)} & V_{(2)}
\end{array}
$$

of any two functions $U$ and $V$ is of frequent occurrence and will be denoted briefly by $\{U, V\}$. Still more frequently occurs the expression $\beta\{U, V\}$,

* This notation is also used for the discriminant of the quadratio differential form in $n$ variables.

† In general throughout this paper, subscripts in parentheses will denote partial differentiation. Thus $U(x, y)$ heing any function of $x$ and $y, U_{(1)}=\partial U / \partial x, U_{(2)}=\partial U / \partial y$. This differs slightly from the notation of MASCHKE, as he used subscripts without parentheses. 
$\beta$ being defined as above; this expression is denoted by $(U, V)$; for our special case, since $\beta=1$, we have $(U, V)=\{U, V\} . *$

If $U$ and $V$ are invariants of the differential form then $(U, V)$ is again an invariant. $†$

3. Differential parameters involving linear.functions. Consider the system of straight lines

parallel to the vector

$$
a(x, y)=a_{1} y-a_{2} x=\text { const. }
$$

$$
a=a_{1} e_{1}+a_{2} e_{2} .
$$

Since $f_{(1)}=e_{1}$ and $f_{(2)}=e_{2}$ we may write $a=(f, a) \cdot \ddagger$

Hence, if $a$ is any linear function, the differential parameter $(f, a)$ may be interpreted as a vector parallel to the system of straight lines $a=$ const.

In particular, $(f, y)=\boldsymbol{e}_{1},(f,-x)=\boldsymbol{e}_{2}$.

Other differential parameters expressed in symbolic form are $(f, a)(f, b)$, $(f, \phi)(f, a),(f, \phi)(f, a)(\phi, b)=(a, b)$, where $a$ and $b$ are functions of $x$ and $y$, and $f$ and $\phi$ are symbolic functions of the differential form. We proceed to find their vector meaning when $a$ and $b$ are linear.

The product $(f, a)(f, b)$, when expanded, equals $a_{1} b_{1}+a_{2} b_{2}$, where as above $a=a_{1} y-a_{2} x$, and $b=b_{1} y-b_{2} x$.

Comparing with the definition, p. 450, we see that this result is the inner product of the two vectors $a=a_{1} e_{1}+a_{2} e_{2}$ and $b=b_{1} e_{1}+b_{2} e_{2}$. The product $(\phi, f)(\phi, a)$, when expanded, equals $-f_{(1)} a_{2}+f_{(2)} a_{1}$. Interpreting $f_{(1)}, f_{(2)}$ as before, we see that this is a vector perpendicular to $\alpha=(f, a)$; with Grassmann we call this the complement $\S$ of the vector $\alpha$ and denote it by $\mid \alpha$; thus

Finally the expression $\|$

$$
(\phi, f)(f, a)=\mid \boldsymbol{a} .
$$

$$
(f, \phi)(f, a)(\phi, b)=(a, b)=a_{1} b_{2}-a_{2} b_{1}
$$

is the so-called outer product $\uparrow$ of $\boldsymbol{a}$ and $\boldsymbol{b}$.

4. Differential parameters involving arbitrary functions. We investigate now the meaning of these same differential parameters when the functions $a$ and $b$ are arbitrary functions of $x$ and $y$.

The equation of the tangent to the curve $a=$ const. at a given point $x_{0}, y_{0}$ on

* The same notation is used in the $n$-dimensional case $;\left\{a_{1}, \cdots, a_{n}\right\}$ represents the Jacobian of the functions $a_{1}\left(x_{1}, \cdots, x_{n}\right), \cdots, a_{n}\left(x_{1}, \cdots, x_{n}\right)$, and $\left(a_{1}, \cdots, a_{n}\right)=\beta\left\{c_{1}, \cdots, a_{n}\right\}$.

$\dagger$ For definitions of "Invariant of a differential quantic," "differential parameter," etc., see Masch Ke, loc. cit., p. 446.

\pm Notice that $a_{(1)}=\partial a / \partial x=-a_{2}$, and $a_{(2)}=\partial a / \partial y=a_{1}$.

$\S$ For the general definition of complement see p. 457.

I Cf. MASCHKE, loo. cit., p. 453, equation (34).

IT The outer product $[a, b]$ of two vectors is usually defined to be $A \cdot B \cdot \sin \hat{a b}$, where $A$ and $B$ are the lengths of $a$ and $b$. This is easily seen to be the same as $a_{1} b_{2}-a_{2} b_{1}$. 
the curve is

or

$$
x\left(\frac{\partial a}{\partial x}\right)_{\substack{x=x_{0} \\ y=y_{0}}}+y\left(\frac{\partial a}{\partial y}\right)_{\substack{x=x_{0} \\ y=y_{0}}}=k=\text { const. }
$$

Let

$$
x\left[a_{(1)}\right]_{\substack{x=x_{0} \\ y=y_{0}}}+y\left[a_{(2)}\right]_{\substack{x=x_{0} \\ y=y_{0}}}=k .
$$

then

$$
L=x\left[a_{(1)}\right]_{\substack{x=x_{0} \\ y=y_{0}}}+y\left[a_{(2)}\right]_{\substack{x=x_{0} \\ y=y_{0}}}
$$

$$
(f, L)=(f, a)_{\substack{x=x_{0} \\ y=y_{0}}}=f_{1}\left[a_{(2)}\right]_{\substack{x=x_{0} \\ y=y_{0}}}-f_{(2)}\left[a_{(1)}\right]_{\substack{x=x_{0} \\ y=y_{0}}}
$$

but, by Art. 3, $(f, L)$ is directed parallel to the straight lines $L=$ const., and these straight lines are parallel to the tangent of that curve of the system $a=$ const. which passes through the point $x_{0}, y_{0}$; hence we have

Theorem I. If the symbols $f_{(1)}, f_{(2)}$ of the differential form $d x^{2}+d y^{2}$ are interpreted as unit vectors along the $x$ - and $y$-axes respectively, then the symbolic differential parameter $(f, a)$ may be interpreted as a vector function of position, tangent at each point of the plane to the curve $a=$ const. which passes through that point.

As in the case where $a$ and $b$ were linear, the product $(f, a)(f, b)$ may be regarded as the inner product of the two vectors represented by $(f, a)$ and $(f, b)$. The expression $(\phi, f)(\phi, a)$ represents a vector perpendicular to $(f, a)$ and therefore normal to the curve $a=$ const.; and the expression $(f, \phi)(f, a)(\phi, b)$ is the outer product of the vectors $(f, a),(f, b)$.

The length* of the vector represented by $(f, a)$ is

$$
\sqrt{(f, a)^{2}}=\sqrt{a_{(1)}^{2}+a_{(2)}^{2}} .
$$

The angle $\theta$ between two curves, $a=$ const., $b=$ const., is given by $\dagger$

or by $\ddagger$

$$
\cos \theta=\frac{(f, a)(f, b)}{\sqrt{(f, a)^{2}} \sqrt{(\phi, b)^{2}}}=\frac{a_{(1)} b_{(1)}+a_{(2)} b_{(2)}}{\sqrt{a_{(1)}^{2}+a_{(2)}^{2}} \sqrt{b_{(1)}^{2}+b_{(2)}^{2}}}
$$

$$
\sin \theta=\frac{(a, b)}{\sqrt{(f, a)^{2}} \sqrt{(\phi, b)^{2}}}=\frac{a_{(1)} b_{(2)}-a_{(2)} b_{(1)}}{\sqrt{a_{(1)}^{2}+a_{(2)}^{2}} \sqrt{b_{(1)}^{2}+b_{(2)}^{2}}} .
$$

5. Change of variables. We obtain greater generality for the plane by changing to curvilinear coördinates. Let us make the substitution

we have then

$$
u=u(x, y), \quad v=v(x, y) ;
$$

$$
d u=u_{(1)} d x+u_{(2)} d y, \quad d v=v_{(1)} d x+v_{(2)} d y .
$$

* Cl. p. 450, second footnote.

†Cl. p. 450 , second footnote.

¥Cl. p. 452, last footnoto. 
Solving for $d x$ and $d y$, we have

$$
d x=\frac{v_{(2)} d u-u_{(2)} d v}{(u, v)}, \quad d y=\frac{u_{(1)} d v-v_{(1)} d u}{(u, v)} .
$$

Substituting these values in the expression for $d s$, we have

$$
d s=f_{(1)} d x+f_{(2)} d y=\frac{(f, v)}{(u, v)} d u+\frac{(u, f)}{(u, v)} d v .
$$

By Theorem I, the coefficients of $d u$ and $d v$ represent vectors tangent at any point $u_{0}, v_{0}$ to the curves $v=$ const., $u=$ const., respectively.

Suppose that the vector function $f(x, y)$ transforms into the vector function $t(u, v)$; we have

$$
f_{(1)}=t_{(u)} u_{(1)}+t_{(v)} v_{(1)}, \quad f_{(2)}=t_{(u)} u_{(2)}+t_{(0)} v_{(2)} .
$$

Solving for $t_{(u)}$ and $t_{(v)}$, we have

$$
t_{(u)}=\frac{(f, v)}{(u, v)}, \quad t_{(v)}=\frac{(u, f)}{(u, v)} .
$$

The quantic giving length of arc

transforms into

$$
d s^{2}=\left(f_{(1)} d x+f_{(2)} d y\right)^{2}=d x^{2}+d y^{2}
$$

$$
\left(t_{(u)} d u+t_{(v)} d v\right)^{2}=E d u^{2}+2 F d u d v+G d v^{2},
$$

where $t_{(u)}^{2}=E, t_{(u)} t_{(v)}=F, t_{(v)}^{2}=G$, so that the function $t(u, v)$ into which $f(x, y)$ transforms is a symbolic function of the transformed differential quantic; hence

Theorem II.* If $A \equiv E d u^{2}+2 F d u d v+G d v^{2}$ is the differential form giving length of arc in a plane, the parameters $u$ and $v$ being arbitrary, then the derivatives $t_{(u)}, t_{(0)}$ of a symbolic function of the form $A$ may be interpreted as vector functions of position, of length $\sqrt{E}, \sqrt{G}$, respectively, tangent at each point of the plane to the parameter curves which pass through that point.

This gives rise to a vector system based on two units,

$$
e_{1}=t_{(u)}, \quad e_{2}=t_{(v)},
$$

tangent to the parameter curves $v=$ const., $u=$ const., respectively; these units are themselves variable from point to point, both in length and in direction.

Again, remembering the factor $\beta=\left(E G-F^{2}\right)^{-\frac{1}{2}}$, we have

$$
(f, a)=(t, a), \quad(f, a)(f, b)=(t, a)(t, b), \dagger \quad \text { etc. }
$$

where the expressions on the right involve derivatives with respect to $x$ and

* Cf. Theorem V.

†Since $(f, a),(f, b)$, etc., are invariant expressions. See MASChKE, loc. cit., p. 449. 
$y$, while the expressions on the left involve derivatives with respect to $u$ and $v$. These expressions, therefore, have always the same geometric interpretation, even when the parameters $u, v$ are changed. In particular, the expression $(t, \tau)(t, a)$, where $t$ and $\tau$ are equivalent symbols of the quantic

$$
E d u^{2}+2 F d u d v+G d v^{2},
$$

still represents the complement of the vector $(t, a)$, so that in this notation the operation of taking the complement is invariant.*

\section{Vectors in Three Dimensions; Axioms.}

6. Fundamental notions. In order to extend the preceding work to space of three dimensions, we shall explain briefly a vector system in that space, following in spirit Grassmann.

Let us start with three mutually perpẹndicular linear vectors, $e_{1}, e_{2}, e_{3}$, which are of unit length and lie along the three axes; equivalence of such linear vectors, their addition and their multiplication follow the rules for two dimensions. The expression $a e_{1}+b e_{2}+c e_{3}$, where $a, b, c$ are ordinary numbers, represents a unique vector defined by these rules, namely, the vector from the origin to the point $a, b, c$, or any parallel vector of equal length ; conversely, any vector in space is expressible in that form.

In order to multiply vectors, we may proceed as in ordinary algebra, provided the fundamental products of $e_{1}, e_{2}, e_{3}$ in pairs are defined; we shall introduce three of these products

$$
E_{1}=\left[e_{2}, e_{3}\right], \quad E_{2}=\left[e_{3}, e_{1}\right], \quad E_{3}=\left[e_{1}, e_{2}\right]
$$

as new units, and call them vectors of the second order. Each may be thought of geometrically as a rectangle of which the factor vectors are sides, or as any equivalent area on any parallel plane. The other products are defined to be

$$
\left[e_{3}, e_{2}\right]=-E_{1}=-\left[e_{2}, e_{3}\right], \quad\left[e_{1}, e_{3}\right]=-E_{2}, \quad\left[e_{2}, e_{1}\right]=-E_{3} .
$$

The products used in (12) and (13) are called outer products. It is to be noted that the area of the rectangles mentioned is equal to the product of the lengths of the sides into the sine of the included angle, - a rule given in two dimensions on p. 452 , footnote. This rule applied to the remaining products gives

$$
\left[e_{1}, e_{1}\right]=\left[e_{2}, e_{2}\right]=\left[e_{3}, e_{3}\right]=0 .
$$

The outer product of any two vectors is obtained in terms of $E_{1}, E_{2}, E_{3}$ by

* The Grassmann definition of complement is given in terms of the units. In general (i. e., except for special transformations) the complement of a vector referred to a new system of units differs from the complement referred to the original units. 
multiplying algebraically the expressions for the two vectors : thus if

then

$$
a=a_{1} e_{1}+a_{2} e_{2}+a_{3} e_{3}, \quad b=b_{1} e_{1}+b_{2} e_{2}+b_{3} e_{3},
$$

$$
[\boldsymbol{a}, \boldsymbol{b}]^{*}=\left(a_{1} b_{2}-a_{2} b_{1}\right) E_{3}+\left(a_{2} b_{3}-a_{3} b_{2}\right) E_{1}+\left(a_{3} b_{1}-a_{1} b_{3}\right) E_{2} \text {. }
$$

It is usual to represent such a sum as occurs in (15) geometrically by a rule for addition of vectors of the second order, similar to the familiar parallelogram law for linear vectors; the parallelogram being replaced by a parallelopiped determined by the two addenda, and the sum being represented by a diagonal parallelogram of the parallelopiped; thus the expression $k_{1} E_{1}+k_{2} E_{2}+k_{3} E_{3}$ may be represented by a certain parallelogram, or by an equivalent area on any parallel plane. It should be noted that the sign of this vector is reversed by reversing the sense in which the perimeter is directed. It follows that the outer product $[a, b]$ of any two linear vectors may be represented by the parallelogram which they determine, with its perimeter taken in the direction shown by $a$.

The outer product of all three units might be represented in a similar fashion, as a vector of the third order; geometrically, a parallelopiped of edges $e_{1}, e_{2}, e_{3}$; it is more usual, however, to notice that all such vectors in space of three dimensions are numerical multiples of one another. It is customary, therefore, to characterize such a vector solely by the number which expresses its volume; we define, then,

$$
\begin{aligned}
& {\left[e_{1}, e_{2}, e_{3}\right]=\left[e_{2}, e_{3}, e_{1}\right]=\left[e_{3}, e_{1}, e_{2}\right]=1,} \\
& {\left[e_{3}, e_{2}, e_{1}\right]=\left[e_{1}, e_{3}, e_{2}\right]=\left[e_{2}, e_{1}, e_{3}\right]=-1 .}
\end{aligned}
$$

The outer product of any three linear vectors can be obtained either by algebraic multiplication and use of $(16)+$ or by computing the volume of the parallelopiped determined by the three vectors; thus if

$$
a=a_{1} e_{1}+a_{2} e_{2}+a_{3} e_{3}, \quad b=b_{1} e_{1}+b_{2} e_{2}+b_{3} e_{3}, \quad c=c_{1} e_{1}+c_{2} e_{2}+c_{3} e_{3},
$$

then

$$
[\boldsymbol{a}, \boldsymbol{b}, \boldsymbol{c}]=\left|\begin{array}{lll}
a_{1} & b_{1} & c_{1} \\
a_{2} & b_{2} & c_{2} \\
a_{3} & b_{3} & c_{3}
\end{array}\right|,
$$

and, using parentheses as equivalent to brackets, we also write

$$
[a, b, c]=([a, b], c)=(a,[b, c]) \text {. }
$$

On occasion, however, we shall distinguish temporarily 3-dimensional vectors from ordinary numbers.

* Cf. definition for two dimensions, p. 452.

$+\Delta$ product in which one of the units is repeated is zero, since the volume of the corresponding parallelopiped is zerb. 
7. Complements; regressive and inner products. Let $E$ be the outer product of several of the unit vectors; we define the complement of $E$, written $\mid E$, to be the outer product of the unit vectors not used in forming $E$, with a sign such that $[E, \mid E]=+1$.

It follows * that $\mid E^{(i)}=\left[E^{(i)}, E^{(3-i)}\right] E^{(3-i)}$, where $E^{(3-i)}$ is the outer product of the unit vectors not used in forming $E^{(i)}$, taken in any order.

The complement of any linear function of several vectors is defined to be the same linear function of their complements ; thus $\dagger$

$$
\left|\left(A_{1} E_{1}+A_{2} E_{2}\right)=A_{1}\right| E_{1}+A_{2} \mid E_{2} .
$$

The outer product of two vectors $A, B$ has been defined only for the case in which the sum of the orders of $A$ and $B$ does not exceed three. In case this sum is greater than three, the sum of the orders of $\mid A$ and $\mid B$ is less than three; hence in this case $[|A| B$,$] is defined. In terms of this product we$ define $[A, B]$.

Definition. If the sum of the orders of $A$ and $B$ exceeds three, then the outer product $[A, B]$ is a vector, $C$, such that $\mid \boldsymbol{C}=[|\boldsymbol{A},| \boldsymbol{B}]$; in this case the product is called regressive; when the sum of the orders of the factors is less than three, the product is called progressive; both are called outer products.

Definition. The outer product $\ddagger[A, \mid B]$ of a vector $A$ and the complement of another vector, $B$, is called the inner product of $A$ and $B$.§

8. Vector formulas. The following formulas enable us to determine the vector represented by a given regressive product. First we consider the regressive product of two units of the second order $\left(\left[e_{i}, e_{j}\right],\left[e_{i}, e_{k}\right]\right)$. We have by definition

$$
\begin{aligned}
\mid\left(\left[\boldsymbol{e}_{i}, \boldsymbol{e}_{j}\right],\left[\boldsymbol{e}_{i}, \boldsymbol{e}_{k}\right]\right) & =\left(\left[\boldsymbol{e}_{i}, \boldsymbol{e}_{j}, \boldsymbol{e}_{k}\right] \boldsymbol{e}_{k},\left[\boldsymbol{e}_{i}, \boldsymbol{e}_{k}, \boldsymbol{e}_{j}\right] \boldsymbol{e}_{j}\right) \\
& =-\left[\boldsymbol{e}_{i}, \boldsymbol{e}_{j}, \boldsymbol{e}_{k}\right]^{2}\left[\boldsymbol{e}_{k}, \boldsymbol{e}_{j}\right]=\left[\boldsymbol{e}_{j}, \boldsymbol{e}_{k}\right] .
\end{aligned}
$$

This by definition is the complement of $\left[e_{i}, e_{j}, e_{k}\right] e_{i}$; hence

$$
\left(\left[e_{i}, e_{j}\right],\left[e_{i}, e_{k}\right]\right)=\left[e_{i}, e_{j}, e_{k}\right] e_{i} \cdot \|
$$

Next consider the product $\left(\left[e_{i}, a\right],\left[e_{i}, e_{k}\right]\right)$ where $a=a_{1} e_{1}+a_{2} e_{2}+a_{3} e_{3}$.

* Capital letters in black face type will be used to denote vectors of uny order. If it is desired to specify the order an upper index in parentheses will be employed ; thus $E^{(i)}$ means a vector of the $i$-th order. Small black face letters invariably represent linear vectors.

$\dagger$ From the definition of complement and formula (18) it follows that $\mid(\mid \boldsymbol{A})= \pm \boldsymbol{A}$.

$\ddagger$ It is customary to omit the comma and regard the complement sign / as the sign of inner multiplication.

\$ This definition, and others of this section are in accord with the corresponding definitions for two dimensions in Art. 2. The definitions as stated here are at once extensible to $n$ dimensions.

$\|$ We have here assumed $i, j, k$ all different, but the formula holds if any two, or if all three are equal, for in that case both sides reduce to zero. 
Substituting this expression for $a$ we have

$$
\begin{aligned}
& \left(\left[\boldsymbol{e}_{i}, \boldsymbol{\alpha}\right],\left[\boldsymbol{e}_{i}, \boldsymbol{e}_{k}\right]\right)=a_{i}\left(\left[\boldsymbol{e}_{i}, \boldsymbol{e}_{i}\right],\left[\boldsymbol{e}_{i}, \boldsymbol{e}_{k}\right]\right)+a_{j}\left(\left[\boldsymbol{e}_{i}, \boldsymbol{e}_{j}\right],\left[\boldsymbol{e}_{i}, \boldsymbol{e}_{k}\right]\right), \\
& \quad+a_{k}\left(\left[\boldsymbol{e}_{i}, \boldsymbol{e}_{k}\right],\left[\boldsymbol{e}_{i}, \boldsymbol{e}_{k}\right]\right)=a_{j}\left(\left[\boldsymbol{e}_{i}, \boldsymbol{e}_{j}\right],\left[\boldsymbol{e}_{k}, \boldsymbol{e}_{i}\right]\right)=\left[\boldsymbol{e}_{i}, \boldsymbol{a}, \boldsymbol{e}_{k}\right] \boldsymbol{e}_{i} .
\end{aligned}
$$

Continuing we find, for any three vectors, $a, b, c$,

$$
([\boldsymbol{a}, \boldsymbol{b}],[\boldsymbol{a}, \boldsymbol{c}])=[\boldsymbol{a}, \boldsymbol{b}, \boldsymbol{c}] \boldsymbol{a} .
$$

Formulas (19) and (20) are special cases of (21).

We are now able to see the geometric meaning of a regressive product $[A, B]$ in case $A$ and $B$ are vectors of the second order; let the planes in which $A$ and $B$ are supposed to lie be produced to meet, and let $a$ be a vector in their line of intersection; then, by properly choosing $\boldsymbol{b}$ and $\boldsymbol{c}$, we may write $A=[\boldsymbol{a}, \boldsymbol{b}]$ and $B=[a, c]$. By $(21)$, then, we see that $[A, B]$ is a vector of the first order lying in (or parallel to) the line of intersection of the planes of $\boldsymbol{A}$ and $\boldsymbol{B}$.*

The inner product of two vectors

$$
\boldsymbol{a}=\sum_{i=1}^{3} a_{i} \boldsymbol{e}_{i}, \quad \boldsymbol{b}=\sum_{i=1}^{3} b_{i} \boldsymbol{e}_{i}
$$

may be found by applying the definition and multiplying out algebraically ; thus

$$
[\boldsymbol{a} \mid \boldsymbol{b}]=\left[\sum_{i=1}^{3} a_{i} \boldsymbol{e}_{i}, \sum_{i=1}^{3} b_{i} \mid \boldsymbol{e}_{i}\right]=\left[\boldsymbol{e}_{1}, \boldsymbol{e}_{2}, \boldsymbol{e}_{3}\right] \sum_{i=1}^{3} a_{i} b_{i}=a_{1} b_{1}+a_{2} b_{2}+a_{3} b_{3} .
$$

This is seen to be the extension to three dimensions of the definition of inner product given on p. 450 .

Again, the inner product $([a, b] \mid c)$ may be found similarly to be

$$
\left(a_{1} b_{2}-a_{2} b_{1}\right)\left(c_{1} e_{2}-c_{2} e_{1}\right)+\left(a_{2} b_{3}-a_{3} b_{2}\right)\left(c_{2} e_{3}-c_{3} e_{2}\right)+\left(a_{3} b_{1}-a_{1} b_{3}\right)\left(c_{3} e_{1}-c_{1} e_{3}\right) \text {. }
$$

If the inner product of this vector and a vector

be formed, the result is

$$
\boldsymbol{d}=\sum_{i=1}^{3} d_{i} \boldsymbol{e}_{i}
$$

$$
\begin{aligned}
\{([\boldsymbol{a}, \boldsymbol{b}] \mid \boldsymbol{c}) \mid \boldsymbol{d}\}= & \left(a_{1} b_{2}-a_{2} b_{1}\right)\left(c_{1} d_{2}-c_{2} d_{1}\right) \\
& +\left(a_{2} b_{3}-a_{3} b_{2}\right)\left(c_{2} d_{3}-c_{3} d_{2}\right)+\left(a_{3} b_{1}-a_{1} b_{3}\right)\left(c_{3} d_{1}-c_{1} d_{3}\right)
\end{aligned}
$$

and this same result is found for the inner product $([a, b] \mid[c, d])$; hence

$$
([\boldsymbol{a}, \boldsymbol{b}] \mid[\boldsymbol{c}, \boldsymbol{d}])=\{([\boldsymbol{a}, \boldsymbol{b}] \mid \boldsymbol{c}) \mid \boldsymbol{d}\} \text {. }
$$

We proceed to obtain certain other fundamental formulas: let $a, b, c$ be any three independent linear vectors, and $\boldsymbol{d}$ any other linear vector. Then we may

* In $n$ dimensions if the proluct $[A, B]$ is progressive, it represents a vector in the least space containing $\boldsymbol{A}$ and $\boldsymbol{B}$; if regressive, a vector in the greatest space common to $\boldsymbol{A}$ and $\boldsymbol{B}$. 
write

$$
\boldsymbol{d}=l \boldsymbol{a}+m \boldsymbol{b}+n \boldsymbol{c},
$$

where $l, m, n$ are ordinary numbers. To obtain $l, m, n$, take the outer product of $\boldsymbol{d}$ with $[\boldsymbol{a}, \boldsymbol{b}],[\boldsymbol{b}, \boldsymbol{c}],[\boldsymbol{c}, \boldsymbol{a}]$, respectively. We thus obtain

$$
[\boldsymbol{a}, \boldsymbol{b}, \boldsymbol{d}]=n[\boldsymbol{\alpha}, \boldsymbol{b}, \boldsymbol{c}],[\boldsymbol{b}, \boldsymbol{c}, \boldsymbol{d}]=l[\boldsymbol{\alpha}, \boldsymbol{b}, \boldsymbol{c}],[\boldsymbol{c}, \boldsymbol{\alpha}, \boldsymbol{d}]=m[\boldsymbol{a}, \boldsymbol{b}, \boldsymbol{c}] ;
$$

hence

$$
l=\frac{[\boldsymbol{b}, \boldsymbol{c}, \boldsymbol{d}]}{[\boldsymbol{a}, \boldsymbol{b}, \boldsymbol{c}]}, \quad m=\frac{[\boldsymbol{c}, \boldsymbol{a}, \boldsymbol{d}]}{[\boldsymbol{a}, \boldsymbol{b}, \boldsymbol{c}]}, \quad n=\frac{[\boldsymbol{a}, \boldsymbol{b}, \boldsymbol{d}]}{[\boldsymbol{a}, \boldsymbol{b}, \boldsymbol{c}]} .
$$

Again, taking the outer product of $d$ with $c$, we have

hence

$$
[\boldsymbol{c}, \boldsymbol{d}]=l[\boldsymbol{c}, \boldsymbol{\alpha}]-m[\boldsymbol{b}, \boldsymbol{c}]
$$

\section{Similarly}

$$
([\boldsymbol{a}, \boldsymbol{b}],[\boldsymbol{c}, \boldsymbol{d}])=-l([\boldsymbol{\alpha}, \boldsymbol{b}],[\boldsymbol{a}, \boldsymbol{c}])+m([\boldsymbol{b}, \boldsymbol{\alpha}],[\boldsymbol{b}, \boldsymbol{c}])
$$

$$
=-l[\boldsymbol{a}, \boldsymbol{b}, \boldsymbol{c}] \boldsymbol{a}-m[\boldsymbol{a}, \boldsymbol{b}, \boldsymbol{c}] \boldsymbol{b} *=[\boldsymbol{a}, \boldsymbol{c}, \boldsymbol{d}] \boldsymbol{b}-[\boldsymbol{b}, \boldsymbol{c}, \boldsymbol{d}] \boldsymbol{a} \text {. }
$$

hence

$$
[\boldsymbol{a}, \boldsymbol{b}]=\frac{l}{m}[\boldsymbol{a}, \boldsymbol{d}]-\frac{n}{m}[\boldsymbol{a}, \boldsymbol{c}]
$$

$$
([a, b],[c, d])=[a, b, d] c-[a, b, c] d .
$$

If we represent the two-dimensional vector $[c, d]$ in $(26)$ as the complement of a linear vector $v$, we have

$$
([\boldsymbol{a}, \boldsymbol{b}] \mid \boldsymbol{v})=[\boldsymbol{a} \mid \boldsymbol{v}] \boldsymbol{b}-[\boldsymbol{b} \mid \boldsymbol{v}] \boldsymbol{a} .
$$

Taking the inner product of both sides of this equation by another vector $w$, and using formula (23), we have

$$
([\boldsymbol{a}, \boldsymbol{b}] \mid[\boldsymbol{v}, \boldsymbol{w}])=[\boldsymbol{a} \mid \boldsymbol{v}][\boldsymbol{b} \mid \boldsymbol{w}]-[\boldsymbol{b} \mid \boldsymbol{v}][\boldsymbol{a} \mid \boldsymbol{w}]=\begin{aligned}
& {[\boldsymbol{a} \mid \boldsymbol{v}],[\boldsymbol{a} \mid \boldsymbol{w}]} \\
& {[\boldsymbol{b} \mid \boldsymbol{v}],[\boldsymbol{b} \mid \boldsymbol{w}]}
\end{aligned}
$$

The vector formulas just given for three dimensions are readily extended to $n$ dimensions. The extensions of formulas (27), (28), and (29) are written down here for reference. They may be proved by methods analogous to those just indicated for three dimensions.

Let $\left[\boldsymbol{a}_{1}, \boldsymbol{a}_{2}, \ldots, \boldsymbol{a}_{k}\right]$ and $\left[\boldsymbol{b}_{1}, \boldsymbol{b}_{2}, \ldots, \boldsymbol{b}_{r}\right]$ be two vectors in a space of $n$ dimensions, of orders $k$ and $r$ respectively, and suppose $k+r>n$, so that the product $\left(\left[\boldsymbol{a}_{1}, \boldsymbol{a}_{2}, \ldots, \boldsymbol{a}_{k}\right],\left[\boldsymbol{b}_{1}, \boldsymbol{b}_{2}, \ldots, \boldsymbol{b}_{i}\right]\right)$ is regressive. The extension of (27) may then be written

$$
\begin{aligned}
\left(\left[\boldsymbol{a}_{1}, \cdots, \boldsymbol{a}_{k}\right],\right. & {\left.\left[\boldsymbol{b}_{1}, \ldots, \boldsymbol{b}_{r}\right]\right) } \\
& =\sum_{c}\left[\boldsymbol{a}_{1}, \ldots, \boldsymbol{a}_{k}, \boldsymbol{b}_{c_{1}}, \ldots, \boldsymbol{b}_{n_{n-k}}\right]\left[\boldsymbol{b}_{c_{n-k-1}}, \ldots, \boldsymbol{b}_{\cdots,}\right] \dagger
\end{aligned}
$$

* Cf. fornula (21), p. 458.

$\dagger$ Ausdehnungslehre, p. 83, no. 113. 
where $c_{1}, \cdots, c_{n-k}$ stands for a combination of $n-k$ of the subscripts $1,2, \ldots, r$, and $c_{1}, c_{2}, \ldots, c_{r}$ are taken in such order that $\left[\boldsymbol{b}_{c_{1}}, \boldsymbol{b}_{c_{9}}, \ldots, \boldsymbol{b}_{c_{r}}\right]$ $=\left[b_{1}, b_{2}, \ldots, b_{r}\right]$. We have also

$$
\begin{aligned}
& \left(\left[a_{1}, a_{2}, \ldots, a_{k+r}\right] \mid\left[b_{1}, b_{2}, \ldots, b_{r}\right]\right) \\
& =\sum_{c}\left(\left[\boldsymbol{a}_{c_{1}}, \cdots, \boldsymbol{a}_{c_{r}}\right] \mid\left[\boldsymbol{b}_{1}, \cdots, \boldsymbol{b}_{r}\right]\right)\left[\boldsymbol{a}_{c_{r+1}}, \cdots, \boldsymbol{a}_{r_{r+1}}\right], * \\
& \left(29_{n}\right) \quad\left(\left[\boldsymbol{a}_{1}, \cdots, \boldsymbol{a}_{k i}\right] \mid\left[\boldsymbol{b}_{1}, \cdots, \boldsymbol{b}_{k}\right]\right)=\left|\begin{array}{c}
{\left[\boldsymbol{a}_{2} \mid \boldsymbol{b}_{1}\right],\left[\boldsymbol{a}_{2} \mid \boldsymbol{b}_{2}\right] \cdots\left[\boldsymbol{a}_{2} \mid \boldsymbol{b}_{k}\right]} \\
\vdots \\
{\left[\boldsymbol{a}_{k} \mid \boldsymbol{b}_{1}\right],\left[\boldsymbol{a}_{k} \mid \boldsymbol{b}_{2}\right] \cdots\left[\boldsymbol{a}_{k} \mid \boldsymbol{b}_{k}\right]}
\end{array}\right| \cdot \dagger
\end{aligned}
$$

9. Axioms. The essential properties $\ddagger$ used in what precedes may be stated in the following axioms, which will be seen to ensure the preceding facts for three dimensions, and which also furnish a sufficient basis for an immediate extension of all these ideas to space of $n$ dimensions for a system of $n$ mutually orthogonal linear unit vectors. The notation explained in footnote, p. 457, will be employed. Small letters in ordinary type denote ordinary numbers.

I. If $\boldsymbol{A}, \boldsymbol{B}, \boldsymbol{C}$ are vectors of the $r$-th order, $r \leqq n$, then

(1) $l A=A l$

(2) $l(m A)=l m A$;

(3) $0 \cdot A=0$;

(4) $l A=0$ implies $l=0$ or $A=0$;

(5) $l A+m B$ is a uniquely determined vector of order $r$;

(6) $A+(B+C)=(A+B)+C$;

(7) $l A+m A=(l+m) A$;

(8) $l(\boldsymbol{A}+\boldsymbol{B})=l \boldsymbol{A}+l \boldsymbol{B} . \S$

II. There exist $n$ vectors of the first order, $e_{1}, e_{2}, \ldots, e_{n}$, such that any linear relation, $l_{1} \boldsymbol{e}_{1}+l_{2} \boldsymbol{e}_{2}+\cdots+l_{n} \boldsymbol{e}_{n}=0$, implies $l_{1}=0, l_{2}=0, \cdots, l_{n}=0$.

Definition. $k$ vectors of any order $A_{1}, A_{2}, \ldots, A_{k}$ are said to be independent if a linear relation, $l_{1} A_{1}+l_{2} A_{2}+\cdots+l_{k} A_{k}=0$, implies $l_{1}=0, l_{2}=0$, $\cdots, l_{k}=0$.

III. If $A^{(i)}$ is a vector of the $i$-th order, and if $B^{(j)}$ and $C^{(j)}$ are vectors of the $j$-th order $(i+j \leqq n)$, then

(1) $\left[A^{(i)}, B^{(S)}\right]$ is a uniquely determined vector of the $(i+j)$-th order ;

(2) $\left[A^{(i)},\left(B^{(j)}+C^{(j)}\right)\right]=\left[A^{(i)}, B^{(j)}\right]+\left[A^{(i)}, C^{(j)}\right]$.

* Ibid., p. 133, no. 173.

† Ibid., p. 135, no. 175.

† This system of axioms is compiled from GrassmanN ; all, however, are not given explioitly by him. Some are given as theorems; others are implied in his definitions. The system, as suoh, is new.

§ The property $A+B=B+A$ can be proved by showing that $(A+B)-(B+A)=0$, by use of $(6),(7),(8)$; hence by $(5)$, the result follows. 
Definition. The expression $\left[A^{(i)}, B^{(f)}\right]$ is called the outer product of $A^{(i)}$ and $B^{(s)}$.

IV. If $\alpha$ and $b$ are vectors of the first order

$$
\begin{aligned}
& {[l \boldsymbol{a}, m \boldsymbol{b}]=\operatorname{lm}[\boldsymbol{a}, \boldsymbol{b}] ;} \\
& {[\boldsymbol{a}, \boldsymbol{b}]+[\boldsymbol{b}, \boldsymbol{a}]=0 .}
\end{aligned}
$$

V. If $a_{1}, a_{2}, \ldots, a_{i}$ and $b_{1}, b_{2}, \ldots, b_{j}$ are vectors of the first order and if $i+j \leqq n$, then

$$
\left(\left[a_{1}, a_{2}, \cdots, a_{i}\right],\left[b_{1}, b_{2}, \cdots, b_{j}\right]\right)=\left[a_{1}, a_{2}, \cdots, a_{i}, b_{1}, b_{2}, \ldots, b_{j}\right] \text {. }
$$

VI. $n$ independent vectors $e_{1}, e_{2}, \cdots, e_{n}$ of the first order exist, such that $\left[e_{1}, e_{2}, \cdots, e_{n}\right]=1$.

Definition. Let $e_{1}, e_{2}, \ldots, e_{n}$ be any $n$ independent vectors of the first order; the products involving the different combinations of these vectors $i$ at a time are called the multiplicative combinations of the $i$-th order of these vectors.

VII. If $A^{(i)}$ is any vector of the $i$-th order, then $n$ independent vectors $e_{1}, e_{2}, \cdots, e_{n}$ of the first order and

$$
N_{i}=\frac{n !}{i !(n-i) !}
$$

numbers, $l_{1}, l_{2}, \cdots, l_{\Lambda_{i}}$, exist such that $A^{(i)}=l_{1} E_{1}+l_{2} E_{2}+\cdots+l_{N_{i}} E_{N_{i}}$, where the $E$ 's are the multiplicative combinations of the $i$-th order of $e_{1}, e_{2}, \cdots, e_{n}$.

Complements, regressive and inner products are defined for $n$ dimensions precisely as on p. 457 for three dimensions. Applying the definition of inner multiplication to the units we $h$ ve

$$
\left[e_{i} \mid e_{j}\right]=\begin{array}{ll}
0 & (i \neq j)^{*} \\
1 & (i=j)
\end{array}
$$

where the units are the $e_{1}, e_{2}, \cdots, e_{n}$ of Axiom VI.

\section{Interpretation of Symbolic Forms and Comparison with the Aхгомs.}

10. Definitions. It will now be shown that symbolic differential parameters of the special form

$$
d x^{2}+d y^{2}+d z^{2}=\left(f_{(1)} d x+f_{(2)} d y+f_{(3)} d z\right)^{2}
$$

may be regarded as vectors of a three-dimensional space by interpreting $f_{(1)}$, $f_{(2)}, f_{(3)}$ as unit vectors along the $x-, y$-, and $z$-axes respectively. This will be done by showing that with suitable definition of outer product, etc., they satisfy the Axioms I-VII for $n=3$.

Linear functions of any number of symbolic parameters of the type $(f, a, b)$, where $f$ is a symbolic function of the differential form (30), will be shown to be

\footnotetext{
* Compare with the formulas for $f(i) f(j)$, footnote, p. 450.
} 
one-dimensional vectors; it should be noted that $f_{(1)}, f_{(2)}, f_{(3)}$ are themselves of this type, since $f_{(1)}=(f, y, z), f_{(2)}=(f, z, x), f_{(3)}=(f, x, y)$.

Also $(f, \phi, \psi)(\phi, \psi, a)$ is a linear function of parameters of this type since, when it is expanded, $\phi$ and $\psi$ disappear according to equations in the footnote, p. 450.

Linear functions of parameters of the type $(f, \phi, a)$ involving two symbolic functions will be shown to be vectors of the second order.

For effecting transformations of certain symbolic expressions, the following determinant theorem, stated for determinants of the third order, is found useful.

Denote

by $|a, b, c|$; then

$$
\begin{array}{lll}
a_{1} & b_{1} & c_{1} \\
a_{2} & b_{2} & c_{2} \\
a_{3} & b_{3} & c_{3}
\end{array}
$$

$|a, b, c| \cdot|d, e, f|=|d, b, c| \cdot|a, e, f|+|e, b, c| \cdot|d l, a, f|+|f, b, c| \cdot|d, e, a| .^{*}$

Let us use the notation

$$
\begin{aligned}
& \boldsymbol{a}=\frac{1}{2}(f, \phi, \psi)(\phi, \psi, a)=a_{(1)} f_{(1)}+a_{(2)} f_{(2)}+a_{(3)} f_{(3)}, \\
& \boldsymbol{b}=\frac{1}{2}(f, \phi, \psi)(\phi, \psi, b)=b_{(1)} f_{(1)}+b_{(2)} f_{(2)}+b_{(3)} f_{(3)}, \\
& \boldsymbol{c}=\frac{1}{2}(f, \phi, \psi)(\phi, \psi, c)=c_{(1)} f_{(1)}+c_{(2)} f_{(2)}+c_{(3)} f_{(3)},
\end{aligned}
$$

where $a, b, c$ are functions of $x, y, z$.

We define outer products as follows :

$$
\begin{gathered}
{[\boldsymbol{a}, \boldsymbol{b}]=\frac{1}{2 !}(f, \phi, \psi)(f, a, b),} \\
{[\boldsymbol{a}, \boldsymbol{b}, \boldsymbol{c}]=([\boldsymbol{a}, \boldsymbol{b}], \boldsymbol{c})=(\boldsymbol{a},[\boldsymbol{b}, \boldsymbol{c}])=(a, b, c) . \dagger}
\end{gathered}
$$

We take Axioms $\operatorname{III}_{(2)}$ and $\mathrm{IV}_{(1)}$ as definitions of the expressions

$$
\left[\boldsymbol{A}^{(i)},\left(\boldsymbol{B}^{(j)}+\boldsymbol{C}^{(j)}\right)\right] \quad \text { and } \quad[l \boldsymbol{a}, m \boldsymbol{b}] \text {. }
$$

11. Comparison with the axioms. We proceed to show that with these definitions Axioms I to VII are satisfied.

* For determinants of the $n$-th order

$$
\left|a_{1}, a_{2}, \cdots, a_{n}\right| \cdot\left|b_{1}, b_{2}, \cdots, b_{n}\right|=\sum_{i=1}^{n}\left|b_{i}, a_{2}, \cdots, a_{n}\right| \cdot\left|b_{1}, b_{2}, \cdots, b_{i-1}, a_{1}, b_{i+1}, \cdots, b_{n}\right| .
$$

See Maschke, Differential purameters of the first order, Transactious of the American Mathematical Society, vol. 7 (1906), p. 70, equation (1). Cf. also E. PASCAL, Lincei Rendiconti, 1888.

$\nmid$ This definition is given in order to conform to the customary definition of $[a, b, c]$ which makes this product a number rather than a vector of the third order. $A$ definition more in accord with the other definitions given here would be

$$
[\boldsymbol{a}, \boldsymbol{b}, \boldsymbol{c}]=(f, \phi, \psi)(\boldsymbol{a}, \boldsymbol{b}, \boldsymbol{c}) / \sqrt{/ \overline{3} !}
$$


$I_{(1)} \ldots I_{(s)}$ are satisfied by the fundamental assumptions in regard to symbols.*

To show that II is satisfied we show that $f_{(1)}, f_{(2)}, f_{(3)}$ are linearly independent. Assume then a linear relation

$$
\eta f_{(1)}+q f_{(2)}+r f_{(3)}=0 ;
$$

multiplying through by $f_{(1)}$, we have

$$
p f_{(1)}^{2}+q f_{(1)} f_{(2)}+r \cdot f_{(1)} f_{(3)}=0 .
$$

But $f_{(1)}^{2}=1, f_{(1)} f_{(2)}=0, f_{(1)} f_{(3)}=0$. Hence the relation reduces to $p=0$. Similarly, multiplying in turn by $f_{(2)}, f_{(3)}$ we find $q=0, r=0$. Hence $f_{(1)}$, $f_{(2)}$, and $f_{(3)}$ may be taken as the $e_{1}, e_{2}$, and $e_{3}$ of II.

$\mathrm{III}_{(2)}$ and $\mathrm{IV}_{(1)}$ are satisfied by definition.

III $_{(1)}$ is satisfied by definition in case the two factors are of the first order; also in case the factors are one of the second order and one of the first order in the forms

$$
\frac{1}{\sqrt{2} !}(f, \phi, \psi)(f, a, b)
$$

and $(f, \phi, \psi)(\phi, \psi, a) / 2$ respectively. There remains the case in which the factors are of the form $(\phi, \psi, a)$ and $(f, \phi, \psi)(\phi, \psi, b)$. In this case, since $(x, y, z)=1$, we may write

$(\phi, \psi, a)=(\phi, \psi, a)(x, y, z)=(\phi, \psi, x)(a, y, z)+(\phi, \psi, y)(a, z, x)+(\phi, \psi, z)(a, x, y)$

by the determinant theorem. Now

$(f, \phi, \psi)=(f, \phi, \psi)(x, y, z)=(f, y, z)(\phi, \psi, x)+(f, z, x)(\phi, \psi, y)+(f, x, y)(\phi, \psi, z)$.

Hence

$(f, \phi, \psi)(f, y, z)=(f, y, z)^{2}(\phi, \psi, x)+(f, z, x)(f, y, z)(\phi, \psi, y)$

$$
+(f, x, y)(f, y, z)(\phi, \psi, z)
$$

and similarly

$$
=f_{(1)}^{2}(\phi, \psi, x)+f_{(1)} f_{(2)}(\phi, \psi, y)+f_{(1)} f_{(3)}(\phi, \psi, z)=(\phi, \psi, x) \text {; }
$$

Hence finally

$$
\begin{aligned}
& (f, \phi, \psi)(f, z, x)=(\phi, \psi, y), \\
& (f, \phi, \psi)(f, x, y)=(\phi, \psi, z) .
\end{aligned}
$$

$(\phi, \psi, a)=(a, y, z) \cdot(f, \phi, \psi)(f, y, z)+(a, z, x) \cdot(f, \phi, \psi)(f, z, x)$

Hence if

$$
+(a, x, y) \cdot(f, \phi, \psi)(f, x, y) \text {. }
$$

$$
\boldsymbol{A}=\frac{1}{1 / 2}(\phi, \psi, a) \quad \text { and } \quad \boldsymbol{B}=\frac{1}{2}(f, \phi, \psi)(\phi, \psi, b),
$$


then

$[A, B]=(a, y, z)(b, y, z)+(a, z, x)(b, z, x)+(a, x, y)(b, x, y)$.

$\mathrm{IV}_{(2)}$ is satisfied by a property of determinants.

$\mathrm{V}$ is satisfied by definition.

VI is satisfied by taking $a, b, c$, equal to $x, y, z$, respectively.

We shall prove that Axiom VII is satisfied by showing that every symbolic differential parameter of the type $(f, a, b)$ can. be expressed linearly in terms of $(f, y, z),(f, z, x),(f, x, y)$ and that every symbolic differential parameter of the type $(f, \phi, a)$ can be expressed linearly in terms of the three parameters $(f, \phi, x),(f, \phi, y),(f, \phi, z)$.

Using the determinant theorem we have

$(f, a, b)=(f, a, b)(x, y, z)=(x, a, b)(f, y, z)+(y, a, b)(f, z, x)$

and also

$$
+(z, a, b)(f, x, y) \text {; }
$$

$$
\begin{aligned}
(f, \phi, a)=(f, \phi, a)(x, y, z)= & (a, y, z)(f, \phi, x) \\
& +(a, z, x)(f, \phi, y)+(a, x, y)(f, \phi, z) .
\end{aligned}
$$

It is shown in the next article that $(f, \phi, x) / \sqrt{2},(f, \phi, y) / \sqrt{2},(f, \phi, z) / \sqrt{2}$ are the multiplicative combinations of the second order of $\boldsymbol{e}_{(1)}=f_{(1)}, \boldsymbol{e}_{(2)}=f_{(2)}$, $e_{(3)}=f_{(s)}$. Axiom VII is, therefore, satisfied if we take

$$
(f, y, z)=f_{(1)}, \quad(f, z, x)=f_{(z)}, \quad(f, x, y)=f_{(3)}
$$

as the $e_{1}, e_{2}, e_{3}$.

The statement made at the head of this section is therefore justified.

The similar work for $n$ dimensions is an easy generalization; hence we have TheOrem III. The symbols $f_{(1)}, f_{(2)}, \cdots, f_{(n)}$, of the special differential form

$$
\sum_{i=1}^{n} d x_{1}^{2}
$$

may be regarded as unit vectors along the coördinate axes ; linear functions of parameters of the type $\left(f_{1}, f_{2}, \ldots, f_{k}, a_{1}, a_{2}, \ldots, a_{n-k}\right)$ involving $k$ symbolic functions $f_{1}, \cdots, f_{k}$, may then be interpreted as $k$-dimensional vectors lying in the space determined by $f_{(1)}, f_{(2)}, \cdots, f_{(n)}$.

In $n$ dimensions we use the notation

$$
\begin{gathered}
a_{i}=\frac{1}{(n-1) !}\left(\phi_{1}, \cdots, \phi_{n-1}, f\right)\left(\phi_{1}, \ldots, \phi_{n-1}, a_{i}\right)=\sum_{j=1}^{n} a_{i(j)} f_{(j)}, \\
b=\frac{1}{(n-1) !}\left(\phi_{1}, \cdots, \phi_{n-1}, f\right)\left(\phi_{1}, \ldots, \phi_{n-1}, b_{i}\right),
\end{gathered}
$$

and define outer products as follows: 


$$
\begin{aligned}
& {\left[a_{1}, a_{2}, \cdots, a_{k}\right]=\frac{1}{(n-k) ! \sqrt{k !}}\left(\phi_{1}, \cdots, \phi_{n-k}, f_{1}, \cdots, f_{k}\right)\left(\phi_{1}, \cdots, \phi_{n-k}, a_{1}, \cdots, a_{k}\right),} \\
& \left(\left[a_{1}, \cdots, a_{k}\right],\left[b_{1}, \cdots, b_{j}\right]\right)=\frac{1}{(n-k-j) ! \sqrt{(k+j) !}} \\
& \times\left(\phi_{1}, \cdots, \phi_{n-k-j}, f_{1}, \cdots, f_{k+j}\right)\left(\phi_{1}, \cdots, \phi_{n-k-j}, a_{1}, \cdots, a_{k}, b_{1}, \cdots, b_{j}\right) \\
& \text { V. FORMULAS AND APPLICATIONS. }
\end{aligned}
$$

12. General formulas. The symbolic expressions for various combinations of vectors will now be obtained and the formulas will then be applied to obtain new relations connecting symbolic differential parameters. We first give different symbolic expressions for the units and their multiplicative combinations.

For,

$$
\begin{aligned}
& e_{1}=\frac{1}{2}(f, \phi, \psi)(\phi, \psi, x)=(f, y, z), \\
& e_{2}=\frac{1}{2}(f, \phi, \psi)(\phi, \psi, y)=(f, z, x), \\
& e_{3}=\frac{1}{2}(f, \phi, \psi)(\phi, \psi, z)=(f, x, y) .
\end{aligned}
$$

$(f, \phi, \psi)=(f, \phi, \psi)(x, y, z)$

and

$$
=(f, y, z)(\phi, \psi, x)+(f, z, x)(\phi, \psi, y)+(f, x, y)(\phi, \psi, z)
$$

since

and

$$
\frac{1}{2}(f, \phi, \psi)(\phi, \psi, x)=\frac{1}{2}\{2(f, y, z)\}=(f, y, z),
$$

$$
(\phi, \psi, x)(\phi, \psi, x)=2
$$

$$
(\phi, \psi, x)(\phi, \psi, y)=(\phi, \psi, x)(\phi, \psi, z)=0 \text {. }
$$

Similarly for the others.

By definition

$$
e_{1}=\frac{1}{2}(f, \phi, \psi)(\phi, \psi, x) .
$$

For the units of the second order we have

$$
\begin{aligned}
& {\left[e_{2}, e_{3}\right]=\mid e_{1}=\frac{1}{\sqrt{2}}(f, \phi, \psi)(f, y, z)=\frac{1}{\sqrt{2}}(\phi, \psi, x),} \\
& {\left[e_{3}, e_{1}\right]=\mid e_{2}=\frac{1}{\sqrt{2}}(f, \phi, \psi)(f, z, x)=\frac{1}{\sqrt{2}}(\phi, \psi, y),} \\
& {\left[e_{1}, e_{2}\right]=\mid e_{3}=\frac{1}{\sqrt{2}}(f, \phi, \psi)(f, x, y)=\frac{1}{\sqrt{2}}(\phi, \psi, z) .}
\end{aligned}
$$

For, by definition, $\mid e_{1}=\left[e_{2}, e_{3}\right]$, where $e_{2}=\frac{1}{2}(f, \phi, \psi)(\phi, \psi, y)$ and $e_{3}=\frac{1}{2}(f, \phi, \psi)(\phi, \psi, z)$ and by definition, p. 462,

$$
\left[e_{2}, e_{3}\right]=\frac{1}{\sqrt{2}}(f, \phi, \psi)(f, y, z) ;
$$


that $(f, \phi, \psi)(f, y, z)=(\phi, \psi, x)$ is proved by writing

$$
(f, \phi, \psi)=(f, \phi, \psi)(x, y, z),
$$

etc., as in the proof of formulas (31).

By this same method it may be shown that

$$
a=\frac{1}{2}(f, \phi, \psi)(\phi, \psi, a)=(a, y, z) e_{1}+(a, z, x) e_{2}+(a, x, y) e_{3}
$$

for $(\phi, \psi, a)=(\phi, \psi, a)(x, y, z)=(\phi, \psi, x)(a, y, z)$, etc., and $\frac{1}{2}(f, \phi, \psi)(\phi, \psi, x)=e_{1}$, etc.

Other important formulas follow :

$$
\mid a=\frac{1}{\sqrt{2}}(\phi, \psi, a) ; *
$$

for by (33)

$$
\begin{aligned}
\mid \boldsymbol{a} & =(a, y, z)\left|\boldsymbol{e}_{1}+(a, z, x)\right| \boldsymbol{e}_{2}+(a, x, y) \mid \boldsymbol{e}_{3} \\
& =\frac{1}{\sqrt{2}}\{(a, y, z)(\phi, \psi, x)+(a, z, x)(\phi, \psi, y)+(a, x, y)(\phi, \psi, z)\},
\end{aligned}
$$

and

$$
\frac{1}{\sqrt{2}}(\phi, \psi, a)=\frac{1}{\sqrt{2}}(\phi, \psi, a)(x, y, z)=\frac{1}{\sqrt{2}}(a, y, z)(\phi, \psi, x),
$$

etc., as above.

$$
[\boldsymbol{a} \mid \boldsymbol{b}]=\frac{1}{2}(f, \phi, a)(f, \phi, b)=(a, y, z)(b, y, z)+(a, z, x)(b, z, x)
$$

Proof. By (33)

$$
+(a, x, y)(b, x, y)=a_{(1)} b_{(1)}+a_{(2)} b_{(2)}+a_{(3)} b_{(3)} \text {. }
$$

$$
\boldsymbol{b}=(b, y, z) \boldsymbol{e}_{1}+(b, z, x) \boldsymbol{e}_{2}+(b, x, y) \boldsymbol{e}_{3}
$$

hence

$$
[\boldsymbol{\alpha} \mid \boldsymbol{b}]=(b, y, z)\left[\boldsymbol{\alpha} \mid \boldsymbol{e}_{1}\right]+(b, z, x)\left[\boldsymbol{a} \mid \boldsymbol{e}_{2}\right]+(b, x, y)\left[a \mid \boldsymbol{e}_{3}\right)
$$

and by definition, p. 462 ,

etc., where

$$
\left[a \mid e_{1}\right]=(a, y, z),
$$

$$
a=\frac{1}{2}(f, \phi, \psi)(\phi, \psi, a) \quad \text { and } \quad \mid e_{1}=\frac{1}{\sqrt{2}}(f, \phi, \psi)(f, y, z) ;
$$

hence

$$
[\boldsymbol{a} \mid \boldsymbol{b}]=(a, y, z)(b, y, z)+(a, z, x)(b, z, x)+(a, x, y)(b, x, y) \text {. }
$$

* Except for a numerical factor the complement of any vector expressed in symbolic form is obtained by multiplying by a factor $(f, \phi, \psi)$ made up wholly of symbolic functions, including the symbolic functions which occur only once in the expression for the vector, or by leaving out such a factor in case it is present. 
Again

$$
\begin{aligned}
(f, \phi, a)(f, \phi, b)= & \{(a, y, z)(f, \phi, x)+(a, z, x)(f, \phi, y) \\
& +(a, x, y)(f, \phi, z)\}\{(b, y, z)(f, \phi, x) \\
& +(b, z, x)(f, \phi, y)+(b, x, y)(f, \phi, z)\} \\
= & 2\{(a, y, z)(b, y, z)+(a, z, x)(b, z, x) \\
& +(a, x, y)(b, x, y)\}
\end{aligned}
$$

and this proves formula (35).

$$
\begin{aligned}
{[\boldsymbol{a}, \boldsymbol{b}] } & =\frac{1}{\sqrt{2}}(f, \phi, \psi)(f, a, b) \\
& =(x, a, b)\left[\boldsymbol{e}_{2}, \boldsymbol{e}_{3}\right]+(y, a, b)\left[\boldsymbol{e}_{3}, \boldsymbol{e}_{1}\right]+(z, a, b)\left[\boldsymbol{e}_{1}, \boldsymbol{e}_{2}\right]
\end{aligned}
$$

The proof is similar to the proof of (33).

for from (36)

$$
\mid[\boldsymbol{a}, \boldsymbol{b}]=[|\boldsymbol{a}| \boldsymbol{b}]=(f, \boldsymbol{a}, \boldsymbol{b}) ;
$$

$\mid[\boldsymbol{a}, \boldsymbol{b}]=(x, a, b) \boldsymbol{e}_{1}+(y, a, b) \boldsymbol{e}_{2}+(z, a, b) \boldsymbol{e}_{3}=(x, a, b)(f, y, z)$

$$
+(y, a, b)(f, z, x)+(z, a, b)(f, x, y)=(f, a, b) \text {. }
$$

$$
([\boldsymbol{a}, \boldsymbol{b}],[\boldsymbol{c}, \boldsymbol{d}])=(f, \phi, \psi)(\phi, a, b)(\psi, c, \vec{a}) .
$$

\section{Proof. By definition,}

$$
\begin{aligned}
\mid([\boldsymbol{a}, \boldsymbol{b}],[\boldsymbol{c}, \boldsymbol{d}])=(|[\boldsymbol{a}, \boldsymbol{b}]|[\boldsymbol{c}, \boldsymbol{d}])= & {\left[\sum(x, a, b) \boldsymbol{e}_{1}, \sum(x, c, d) \boldsymbol{e}_{1}\right] } \\
=\left|\begin{array}{l}
(x, a, b),(x, c, d) \\
(y, a, b),(y, c, d)
\end{array}\right|\left[\boldsymbol{e}_{1}, \boldsymbol{e}_{2}\right] & +\left|\begin{array}{l}
(y, a, b),(y, c, d) \\
(z, a, b),(z, c, d)
\end{array}\right|\left[\boldsymbol{e}_{2}, e_{3}\right] \\
+ & +\left|\begin{array}{l}
(z, a, b),(z, c, d) \\
(x, a, b),(x, c, d)
\end{array}\right|\left[\boldsymbol{e}_{3}, \boldsymbol{e}_{1}\right]
\end{aligned}
$$

again,

$(f, \phi, \psi)(\phi, a, b)(\psi, c, d)=(\phi, a, b)\{(f, \phi, x)(\psi, y, z)(\psi, c, d)$

$$
\begin{array}{r}
+(f, \phi, y)(\psi, z, x)(\psi, c, d)+(f, \phi, z)(\psi, x, y)(\psi, c, d)\} \\
=(\phi, a, b)\{(f, \phi, x)(x, c, d)+(f, \phi, y)(y, c, d)+(f, \phi, z)(z, c, d)\} .
\end{array}
$$

Now

$$
\begin{aligned}
& (f, \phi, x)(\phi, a, b)=(f, y, x)(\phi, z, x)(\phi, a, b) \\
& \quad+(f, z, x)(\phi, x, y)(\phi, a, b)=(y, a, b)(f, y, x)+(z, a, b)(f, z, x),
\end{aligned}
$$

with similar expressions for $(f, \phi, y)(\phi, a, b)$ and $(f, \phi, z)(\phi, a, b)$. Sub- 
stituting, we have finally

$$
\begin{aligned}
\cdot(f, \phi, \psi)(\phi, a, b)(\psi, c, d)=\{(y, a, b)(f, y, x) \\
\quad+(z, a, b)(f, z, x)\}(x, c, d)+\{(z, a, b)(f, z, y)+(x, a, b)(f, x, y)\}(y, c, d) \\
\quad+\{(x, a, b)(f, x, z)+(y, a, b)(f, y, z)\}(z, c, d) \\
=(f, x, y)\left|\begin{array}{l}
(x, a, b),(x, c, d) \\
(y, a, b),(y, c, d)
\end{array}\right|+(f, y, z)\left|\begin{array}{l}
(y, a, b),(y, c, d) \\
(z, a, b),(z, c, d)
\end{array}\right| \\
\quad+(f, z, x)\left|\begin{array}{l}
(z, a, b),(z, c, d) \\
(x, a, b),(x, c, d)
\end{array}\right| .
\end{aligned}
$$

The expression found above for $\mid([a, b],[c, d])$ is clearly the complement of this final expression for $(f, \phi, \psi)(\phi, a, b)(\psi, c, d)$; hence the formula is proved.

$$
([\boldsymbol{a}, \boldsymbol{b}] \mid \boldsymbol{c})=(f, \phi, c)(\phi, a, b) .
$$

For by (33) or (34)

hence

$$
\mid c=\Sigma(c, y, z)\left[e_{2}, e_{3}\right]
$$

$$
\begin{aligned}
&([a, b] \mid c)=(c, y, z)\left([a, b],\left[e_{2}, e_{3}\right]\right)+(c, z, x)\left([a, b],\left[e_{3}, e_{1}\right]\right) \\
&+(c, x, y)\left([a, b],\left[e_{1}, e_{2}\right]\right) \\
&=(\phi, a, b) \Sigma(c, y, z)(f, \phi, \psi)(\psi, y, z) \quad \text { by }(38), \\
&=(\phi, a, b) \Sigma(f, \phi, x)(c, y, z)=(f, \phi, c)(\phi, a, b) . \\
&(40) \quad([a, b] \mid[c, d])=(f, a, b)(f, c, d) . *
\end{aligned}
$$

For, by formula (37), $\mid[c, d]=\Sigma(x, c, d) e_{1}$; hence

$$
\begin{aligned}
& ([\boldsymbol{a}, \boldsymbol{b}]] \mid[\boldsymbol{c}, \boldsymbol{d}]) \\
& \quad=(x, c, d)\left[\boldsymbol{a}, \boldsymbol{b}, \mathrm{e}_{1}\right]+(y, c, d)\left[\boldsymbol{a}, \boldsymbol{b}, \mathrm{e}_{2}\right]+(z, c, d)\left[\boldsymbol{a}, \boldsymbol{b}, \mathrm{e}_{3}\right] \\
& \quad=(x, c, d)(x, a, b)+(y, c, d)(y, a, b)+(z, c, d)(z, a, b) ;
\end{aligned}
$$

and $(f, a, b)(f, c, d)$ expanded gives the same.

We give below the extensions of the formulas (31)-(40) to $n$ dimensions; the proofs are similar to the proofs given above for three dimensions.

$$
\begin{aligned}
\boldsymbol{e}_{1} & =\frac{1}{(n-1) !}\left(f, \phi_{1}, \ldots, \phi_{n-1}\right)\left(\phi_{1}, \ldots, \phi_{n-1}, x_{i}\right) \\
& =\left(x_{1}, \ldots, x_{i-1}, f, x_{i+1}, \ldots, x_{n}\right) .
\end{aligned}
$$

* Except for a numerical factor the inner product of two vectors in symbolio notation is obtained by writing them both (or their complements) in terms of the same symbolic functions as tar as possible. 


$$
\begin{aligned}
& {\left[e_{c_{1}}, \cdots, e_{c_{k}}\right]=\frac{1}{(n-k) ! \sqrt{k !}} F_{1} F_{2}=\frac{1}{\sqrt{k !}}\left(f_{1}, \cdots, f_{k}, x_{c_{k+1}}, \cdots, x_{c_{k}}\right),} \\
& F_{1}=\left(f_{1}, \cdots, f_{k}, \phi_{1}, \cdots, \phi_{n-k}\right), \quad F_{2}=\left(\phi_{1}, \cdots, \phi_{n-k}, x_{c_{1}}, \cdots, x_{c_{k}}\right),
\end{aligned}
$$

where $c_{1}, \ldots, c_{k}$ stands for any combination of $k$ of the subscripts $1,2, \ldots, n$, and $c_{k+1}, \cdots, c_{n}$ are the remaining subscripts in such order that $\left(x_{c_{1}}, x_{c_{z}}, \cdots, x_{c_{n}}\right)=+1$.

$$
a=\frac{1}{(n-1) !}\left(f, \phi_{1}, \cdots, \phi_{n-1}\right)\left(\phi_{1}, \cdots, \phi_{n-1} a\right)
$$

$$
\sum_{i=1}^{n}\left(x_{1}, \cdots, x_{i-1}, a, x_{i+1}, \cdots, x_{n}\right) e_{i} .
$$

$$
\mid a=\frac{1}{\sqrt{(n-1) !}}\left(f_{1}, \cdots, f_{n-1}, a\right) .
$$

$$
[a \mid b]=\frac{1}{(n-1) !}\left(f_{1}, \cdots, f_{n-1}, a\right)\left(f_{1}, \cdots, f_{n-1}, b\right) .
$$

$\left[a_{1}, a_{2}, \cdots, a_{k}\right]$

$$
\begin{aligned}
& =\frac{1}{(n-k) ! \sqrt{k !}}\left(\phi_{1}, \ldots, \phi_{n-k}, f_{1}, \cdots, f_{k}\right)\left(\phi_{1}, \cdots, \phi_{n-k}, a_{1}, \cdots, a_{k}\right) \\
& =\sum_{c}\left(a_{1}, \cdots, a_{k}, x_{c_{1}}, \cdots, x_{c_{m+n}}\right)\left[e_{c_{m+n+1}}, \cdots, e_{c_{n}}\right] \text {. } \\
& \mid\left[a_{1}, \ldots, a_{k}\right]=\left(\left|\left[a_{1}, \cdots, a_{i}\right]\right|\left[a_{i+1}, \ldots, a_{k}\right]\right) \\
& =\frac{1}{\sqrt{(n-k) !}}\left(f_{1}, \ldots, f_{n-k}, a_{1}, \cdots, a_{k}\right) \text {. }
\end{aligned}
$$

If $k+j>n$,

$$
\left(\left[a_{1}, \cdots, \alpha_{k}\right],\left[b_{1}, \cdots, b_{j}\right]\right)=\frac{1}{(n-k) !(n-j) ! \sqrt{(k+j-n) !}} M_{1} M_{2},
$$

$$
\begin{aligned}
& M_{1}=\left(\phi_{1}, \cdots, \phi_{n-k}, \psi_{1}, \ldots, \psi_{n-j}, f_{1}, \cdots, f_{k+j-n}\right), \\
& M_{2}=\left(\phi_{1}, \cdots, \phi_{n-k}, a_{1}, \cdots, a_{k}\right)\left(\psi_{1}, \cdots, \psi_{n-j}, b_{1}, \ldots, b_{j}\right) .
\end{aligned}
$$

$\left(39^{n}\right)$

$$
\left(\left[a_{1}, \cdots, a_{k+r}\right] \mid\left[b_{1}, \cdots, b_{r}\right]\right)=\frac{1}{(n-k-r) ! \sqrt{k !}} M_{3} \text {, }
$$$$
M_{3}=\left(f_{1}, \cdots, f_{k}, \phi_{k+1}, \cdots, \phi_{n-r}, b_{1}, \cdots, b_{r}\right)\left(\phi_{k+1}, \cdots, \phi_{n-r}, a_{1}, \cdots, a_{k+r}\right),
$$$$
\left(\left[a_{1}, \cdots, a_{r}\right] \mid\left[b_{1}, \cdots, b_{r}\right]\right)
$$

$$
=\frac{1}{(n-r) !}\left(\phi_{1}, \ldots, \phi_{n-r}, a_{1}, \cdots, a_{r}\right)\left(\phi_{1}, \ldots, \phi_{n-r}, b_{1}, \cdots, b_{r}\right) .
$$


13. Application to the symbolic invariant theory. Results of the Grassmann theory may be employed to derive formulas in the symbolic theory. Three examples of this application are given below.*

1. From formula $\left(27_{n}\right)$ follows by use of formula $\left(38_{n}\right)$

$$
\begin{aligned}
\left(f_{1}, \cdots, f_{n-k}, \phi_{1}, \cdots, \phi_{n-j}, \psi_{1}, \cdots, \psi_{k+j-1}\right)\left(f_{1}, \cdots, f_{n-k}, a_{1}, \cdots, a_{k}\right) \\
\quad \times\left(\phi_{1}, \cdots, \phi_{n-j}, b_{1}, \cdots, b_{j}\right)=\frac{(n-k) !(n-j) !}{(2 n-k-j) !} \\
\quad \times \sum_{c}\left(f_{1}, \cdots, f_{n-k}, \phi_{1}, \cdots, \phi_{n-j}, \psi_{1}, \cdots, \psi_{k+j-n}\right) \\
\quad \times\left(f_{1}, \cdots, f_{n-k}, \phi_{1}, \cdots, \phi_{n-j}, b_{c_{1}}, \cdots, b_{c_{k+j-n}}\right)\left(a_{1}, \cdots, a_{k}, b_{c_{1}}, \cdots, b_{c^{\prime}-k}\right) \cdot \dagger
\end{aligned}
$$

2. From formula $\left(28_{n}\right)$ follows by use of formula $\left(39_{n}\right)$

$$
\begin{aligned}
& \left(f_{1}, \cdots, f_{k}, \phi_{k+1}, \cdots, \phi_{n-r}, b_{1}, \cdots, b_{r}\right)\left(\dot{\phi}_{k+1}, \cdots, \phi_{n-r}, a_{1}, \cdots, a_{k+r}\right) \\
& =\frac{(n-k-r) !}{(n-k) !(n-r) !} \sum_{c}\left(f_{1}, \cdots, f_{n-r}, a_{c_{1}}, \cdots, a_{c_{r}}\right)\left(f_{1}, \cdots, f_{n-r}, b_{1}, \cdots, b_{r}\right) \\
& \quad \times\left(\phi_{1}, \cdots, \phi_{n-k}, a_{c_{1}}, \cdots, a_{c_{k}}\right)\left(\phi_{1}, \cdots, \phi_{n-k}, \psi_{1}, \cdots, \psi_{k}\right) .
\end{aligned}
$$

3. From formula $\left(29_{n}\right)$ follows by use of formulas $\left(35_{n}\right)$ and $\left(40_{n}\right)$

$$
\begin{aligned}
& \frac{1}{(n-k) !}\left(f_{1}, \ldots, f_{n-k}, a_{1}, \ldots, a_{k}\right)\left(f_{1}, \ldots, f_{n-k}, b_{1}, \ldots, b_{k}\right) \\
& =\frac{1}{\{(n-1) !\}^{k}}\left|\begin{array}{c}
\left(f_{1}, a_{1}\right)\left(f_{1}, b_{1}\right),\left(f_{1}, a_{1}\right)\left(f_{1}, b_{2}\right), \ldots,\left(f_{1}, a_{1}\right)\left(f_{1}, b_{k}\right) \\
\left(f_{2}, a_{2}\right)\left(f_{2}, b_{1}\right),\left(f_{2}, a_{2}\right)\left(f_{2}, b_{2}\right), \ldots,\left(f_{2}, a_{2}\right)\left(f_{2}, b_{k}\right) \\
\cdot \cdot \cdot \cdot \cdot \cdot \cdot \cdot \cdot \cdot \cdot \cdot \cdot \cdot \cdot \cdot \\
\left(f_{k}, a_{k}\right)\left(f_{k}, b_{1}\right),\left(f_{k}, a_{k}\right)\left(f_{k}, b_{2}\right), \ldots,\left(f_{k}, a_{k}\right)\left(f_{k}, b_{k}\right)
\end{array}\right|,
\end{aligned}
$$

where $\left(f_{i}, a_{i}\right)=\left(f_{i 1}, f_{i 2}, \cdots, f_{i n-1}, a_{i}\right)$ and $f_{i j}$ is a symbolic function equivalent to $f$.

These formulas can be shown to hold for the general differential form.

14. Applications to geometry. As heretofore, we regard the variables $x_{1}, x_{2}, \ldots, x_{n}$ as cartesian coördinates of a point in an $n$-dimensional space; we wish to determine how the $k$-dimensional vector $\left(f_{1}, \ldots, f_{k}, a_{1}, \ldots, a_{n-k}\right)$ is related to the surfaces $a_{1}=$ const., $a_{2}=$ const., $\cdots, a_{n-l i}=$ const.

First we show that the $(n-1)$-dimensional vector $\left(f_{1}, \cdots, f_{n-1}, a\right)$ is, at

* Formulas (41) and (42) are new.

For formula (43) see MASchke, Differential parameters of the first order, loo. cit., p. 73.

$\dagger c_{1}, \cdots, c_{k+j-n}$ stands for any combination of $k+j-n$ of the subscripts, $1,2, \cdots, j$; $c_{1}^{\prime}, \cdots, c_{n-k}^{\prime}$ are the remaining subscripts. In order to obtain the correct sign in each term, the order of the subscripts must be such that $c_{1}, \cdots, c_{k+j-n}, c_{1}^{\prime}, \cdots, c_{n-k}^{\prime}$ may be obtained from $1,2, \cdots, j$, by an even number of transpositions. 
each point of space, tangent to one of the surfaces $a=$ const. ; for when $a$ is linear, by formulas $\left(33_{n}\right)$ and $\left(34_{n}\right)$,

$$
\frac{1}{\sqrt{(n-1) !}}\left(f_{1}, \cdots, f_{n-1}, a\right)=\sum_{i=1}^{n} a_{(i)} e_{i},
$$

is parallel to the planes $a=$ const.

When $a$ is not linear, then the linear space of $n-1$ dimensions, tangent at a given point $\left(\bar{x}_{1}, \bar{x}_{2}, \ldots, \bar{x}_{n}\right)$ to the surface $a=$ const. which passes through that point, is given by

and clearly

$$
L \equiv \sum_{i=1}^{n}\left(a_{(i)}\right)_{x=. x} x_{i}=\text { const. }
$$

$$
\left(f_{1}, \cdots, f_{n-1}, a\right)_{x=\bar{x}}=\left(f_{1}, \cdots, f_{n-1}, L\right) .
$$

The linear space tangent to the intersection of $a_{1}=$ const., $a_{2}=$ const. will be the space common to the linear spaces tangent to the two hyper-surfaces. A vector lying in this space is found by taking the outer product of the vectors $\left(f_{1}, \cdots, f_{n-1}, a_{1}\right)$ and $\left(f_{1}, \cdots, f_{n-1}, a_{2}\right)$. By formula $\left(37_{n}\right)$ this product may be written, except for a numerical factor, $\left(f_{1}, \cdots, f_{n-2}, a_{1}, a_{2}\right)$.

This argument can be repeated for three functions $a_{1}, a_{2}, a_{3}$, and again, for any number, $a_{1}, a_{2}, \ldots, a_{n-l i}$; hence we have:

TheOREM IV. If the derivatives $f_{(1)}, f_{(2)}, \cdots, f_{(n)}$ of a symbolic function of the differential form

$$
\sum_{i=1}^{n} d x_{i}^{2}
$$

are interpreted as unit vectors along the coördinate axes, then

$$
\left(f_{1}, f_{2}, \cdots, f_{k}, a_{1}, a_{2}, \cdots, a_{n-k}\right)
$$

may be interpreted as a $k$-dimensional vector, tangent to the $k$-dimensional spread which is common to $a_{1}=$ const., $a_{2}=$ const.,..,$a_{n-k}=$ const.

\section{The General Differential Form.}

15. Change of variables in three dimensions. So far we have been considering differential parameters of a special type of differential form : namely, the differential form for length of arc in an Euclidean space; i. e., a form of the type

$$
\sum_{i=1}^{n} d x_{i}^{2}
$$

or any form into which such a form can be transformed.

In this section it is shown that Theorem IV can be extended to apply to any space which can be considered a part of an Euclidean space (of higher dimensions); for example, to a surface lying in three dimensions. 
Let us consider in an ordinary space of three dimensions the transformation

$$
u=u(x, y, z), \quad v=v(x, y, z), \quad w=w(x, y, z) .
$$

We can find $d x, d y, d z$, in terms of $d u, d v, d w$, by solving the equations

$$
\begin{aligned}
d u & =u_{(1)} d x+u_{(2)} d y+u_{(3)} d z, \\
d v & =v_{(1)} d x+v_{(2)} d y+v_{(3)} d z, \\
d w & =w_{(1)} d x+w_{(2)} d y+w_{(3)} d z .
\end{aligned}
$$

If the resulting values are substituted in

we obtain

$$
d s=f_{(1)} d x+f_{(2)} d y+f_{(3)} d z
$$

$$
d s=\frac{(f, v, w)}{(u, v, w)} d u+\frac{(u, f, w)}{(u, v, w)} d v+\frac{(u, v, f)}{(u, v, w)} d w .
$$

By Theorem IV the coefficients* of $d u, d v, d w$ may be regarded as linear vectors tangent to the intersections of $v=$ const., $w=$ const.; $w=$ const., $u=$ const.; and $u=$ const., $v=$ const., respectively. Let us denote these coefficients by $t_{(1)}, t_{(2)}, t_{(3)}$. It can be shown precisely as in the two-dimensional case that $t_{(1)}, t_{(2)}, t_{(3)}$ are the derivatives with respect to $u, v, w$ of the function into which the symbolic function $f$ transforms. Hence the transformed function $t(u, v, w)$ is a symbolic function of the differential quantic into which $d x^{2}+d y^{2}+d z^{2}$ transforms and the derivatives $t_{(u)}, t_{(v)}, t_{(x)}\left(\right.$ i. e., $\left.t_{(1)}, t_{(2)}, t_{(s)}\right)$ may be interpreted as vector functions of position, tangent at each point of space to the parameter curves which pass through that point. The transformed differential form is given by $d s^{2}=d f^{2}=d t^{2}=\left(t_{(1)} d u+t_{(2)} d v+t_{(3)} d w\right)^{2}$ where $t_{(1)}=(f, v, w) /(u, v, w)$, etc.

16. The general two-dimensional case. On the surface $w=a$ (a definite const.), $d w=0$. In our formula for $d s$ let us write $a$ for $w$ to indicate that only such values of $x, y, z$ are to be used as will satisfy the equation $w=a$.

We have, then, on the surface

where

$$
d s^{2}=\left(t_{(1)} d u+t_{(2)} d v\right)^{2}=E d u^{2}+2 F d u d v+G d v^{2}
$$

$$
t_{(1)}^{2}=E, \quad t_{(1)} t_{(2)}=F, \quad t_{(2)}^{2}=G \cdot \dagger
$$

This gives rise to a vector system on a surface, having variable units $t_{(1)}, t_{(2)}$ which are tangent to the parameter curves of the surface. The usual vector expressions, as inner and outer products, and complements can be set up in terms

* Observe that the common denominator of these coeffioients is an ordinary number, being the Jacobian of the functions $u, v, w$ with respect to $x, y, z$.

tThe function $f=f_{(1)} \cdot x+f_{(2)} \cdot y+f_{(3)} \cdot z$ represents the rector from the origin to the point $x, y_{2} z$; hence $t(u, v)$ represents the veotor from the origin to any point on the surface $w=a$. 
of these variable units; these expressions take the same form as the corresponding expressions in the plane given on p. 453.

It should be noticed that here, since $w=a$ is an arbitrary surface, we are considering a perfectly general binary quadratic differential form; hence

Theorem V. If $A \equiv E d u^{2}+2 F d u d v+G d v^{2}$ is any binary quadratic differential form, the partial derivatives $\partial t / \partial u, \partial t / \partial v$ of a symbolic function of $A$ may be interpreted as vectors, of length $\sqrt{E}, \sqrt{G}$ respectively, tangent to the parameter curves of the surface characterized by $A$.

17. The general k-dimensional case. In the same way we consider in an Euclidean space of $n$ dimensions, the sub-space of $k$ dimensions determined by $a_{1}=$ const., $\cdots, a_{n-k}=$ const. For arc length in the sub-space we shall have $d s^{2}=\left(\Sigma t_{(i)} d u_{i}\right)^{2}$, where

$$
t_{(t)}=\frac{\left(u_{1}, u_{2}, \cdots, u_{i-1}, f, u_{i+1}, \cdots, u_{k}, a_{1}, \ldots, a_{n-k}\right)}{\left(u_{1}, \cdots, u_{k}, a_{1}, \cdots, a_{n-k}\right)}
$$

and where $!\left(u_{1}, \ldots, u_{k}\right)=f\left(x_{1}, \ldots, x_{n}\right)$.

Precisely as in the three-dimensional cases it can be shown that $t_{(i)}=\partial t / \partial u_{i}$; hence we have

Theorem VI. If

$$
A \equiv \sum_{i, j=1}^{k} E_{i j} d u_{i} d u_{j}
$$

is the differential form giving length of arc in any space of $k$ dimensions belonging to an. Euclidean space of $n$ dimensions, then the partial derivatives $\partial t / \partial u_{i}, \cdots, \partial t / \partial u_{k}$ of $a$ symbolic function of $A$ may be interpreted as vectors tangent to the parameter curves of the sub-space characterized by $A$.

\section{Symbolic Differential Parameters involving Derivatives of the Second and Higher Orders.}

18. Interpretation of the higher derivatives of the symbols. Let the space $R_{n}$ whose arc element is determined by

$$
d s^{2}=\sum_{i, j=1}^{n} E_{i j} d u_{i} d u_{j}=\left(\sum_{i=1}^{n} f_{(i)} d u_{i}\right)^{2}
$$

be supposed to lie in an Euclidean space $S_{r}$ of $r$ dimensions. The $f_{(i)}$ may then be interpreted as vectors in $S_{r}$ tangent to $R_{n}$ along the parameter curves. Since these tangent vectors lie in $S_{r}$, they may be expressed as linear functions of $r$ constant, mutually orthogonal unit vectors, $e_{1}, e_{2}, \cdots, e_{r}$, in $S_{r}$, the coefficients being functions of $u_{1}, \ldots, u_{n}$. The derivatives of the $f_{(i)}$ of any order, with respect to the variables $u_{1}, u_{2}, \cdots, u_{n}$, if they do not vanish, are again linear functions of the unit vectors, $e_{1}, e_{2}, \ldots, e_{r}$. We have therefore 
Theorem VII. If the space $R_{n}$ whose arc element is determined by

$$
d s^{2}=\sum_{i, j=1}^{n} E_{i j} d u_{i} d u_{j}=\left(\sum_{i=1}^{n} f_{(i)} d u_{i}\right)^{2}
$$

lies in an Euclidean space $S_{r}$ of $r$ dimensions, then the derivatives of the symbolic function $f$, of all orders, with respect to any of the variables $u_{1}, \ldots, u_{n}$, may be interpreted as vectors of the space $S_{r}$.

Since symbolic differential parameters involving derivatives of the $E_{i j}$ to any order are expressible in terms of derivatives of the symbolic functions, it foliows that all such differential parameters may be interpreted as vectors or as combinations of vectors of the space $S_{r}$.

19. Second covariantive derivatives of the symbolic functions. The second covariantive derivatives of the symbolic functions are given linearly in terms of the ordinary second derivatives and of the first derivatives by the formula

$$
f^{(i)}=f_{(i j)}-\frac{1}{(n-1) !} \phi_{(i j)}(\phi, \psi)(f, \psi)^{*} \quad\left(f_{(i j)}=\frac{\partial^{2} f}{\partial u_{i} \partial u_{j}}\right) .
$$

It follows that $f^{(i j)}$ may be interpreted as a vector. But we have always $f_{(k)} f^{(i j)}=0 \dagger$ which shows that each $f^{(i)}$ is orthogonal to all the vectors $f_{(i)}$. Hence

Theorem VIII. If the $f_{\left(k_{i}\right)}$ are interpreted as vectors in $S_{r}$ tangent to $R_{n}$ along the parameter curves, then the second covariantive derivatives $f^{(i)}$ may be interpreted as normals to $R_{n}$ in $S_{r}$.

* M $\Lambda$ SCHKE, A symbolic trentment, etc., loc. cit., p. 457, equation (75).

† MASchKe, loc. cit., p. 459, equation (84). 